\title{
Diagnostics of Adult Forms of Celiac Disease: More Intensive Screening Improves Diagnosis
}

\author{
Peter Makovicky ${ }^{1, *}$, Csaba Podlupszki ${ }^{2}$, Pavol Makovicky ${ }^{3}$, Gabriel Samasca ${ }^{4}$, Alexandra Rejhova ${ }^{5}$, \\ Hugh James Freeman ${ }^{6}$ \\ ${ }^{1}$ Czech Centre for Phenogenomics, Institute of Molecular Genetics, Department of Transgenic Models of Diseases, ASCR, Prague, \\ Czech Republic \\ ${ }^{2}$ Department of Gastroenterology, ForLife - General Hospital in Komarno, Slovak Republic \\ ${ }^{3}$ Department of Biology, Pedagogical Faculty, Selye Janos University, Bratislavska, Slovak Republic \\ ${ }^{4}$ Department of Immunology, Iuliu Hatieganu University of Medicine and Pharmacy, Cluj-Napoca, Romania \\ ${ }^{5}$ Department of the Molecular Biology of Cancer, Institute of Experimental Medicine of the ASCR, Prague, Czech Republic \\ ${ }^{6}$ Department of Medicine (Gastroenterology), University of British Columbia, Vancouver, BC, Canada \\ *Corresponding author: pmakovicky@email.cz
}

\section{Received May 01, 2015; Revised May 10, 2015; Accepted June 10, 2015}

\begin{abstract}
Diagnosis of adult celiac disease is often a problematic part of clinical medicine. High risk groups for adult celiac disease deserve an intense search, even if atypical extra-intestinal symptoms suggest some other underlying cause. In part, diagnosis may occur over a long time period during adult years, and even then, could potentially remain undiagnosed until very late, even in very elderly stages of life. The objective of this work was to record our clinical experience with the diagnosis of adult forms of the celiac disease, in spite of their atypical presentations with extra-intestinal symptoms alone, and consider the role of serological testing for case-finding in these patients. We chose 9 adult patients with celiac disease diagnosed over a long period during an outpatient medical care by various physician specialists. Examination of autoantibodies for tissue transglutaminase, antiendomysium antibodies and autoantibodies against gliadin as well as cow's milk were done. To confirm positive autoantibodies as an indicator of adult celiac disease, samples of duodenal mucosa were taken and histopathologically examined.
\end{abstract}

Keywords: celiac disease, diagnostics, adult patients, screening, hidden forms

Cite This Article: Peter Makovicky, Csaba Podlupszki, Pavol Makovicky, Gabriel Samasca, Alexandra Rejhova, and Hugh James Freeman, "Diagnostics of Adult Forms of Celiac Disease: More Intensive Screening Improves Diagnosis.” International Journal of Celiac Disease, vol. 3, no. 3 (2015): 95-97. doi: 10.12691/ijcd-3-3-1.

\section{Introduction}

Celiac disease (CD) is associated with intolerance to gluten found in various types of cereals and other food products. The "gold standard" diagnostic test is a bioptic examination of a duodenal mucosal sample showing characteristic histopathological features of untreated CD $[1,2,3]$. The diagnostic tests remain within the realm and competence of gastroenterologists [4]. In clinical practice, several clinical or sub-clinical forms of CD may occur with classical or typical forms representing an estimated 40\%. Numerous domestic and foreign publications indicate that the disease is still under-diagnosed $[5,6]$. Atypical forms have been noted, despite very limited intestinal symptoms, such as diarrhoea. Due to these facts there have already been proposed several possibilities in past which would ultimately help reveal atypical forms of $\mathrm{CD}$ in a population of children and adults. Adult forms of the disease are often symptomatically highly variable. This variability of forms suggests a significant challenge to a wider group of professionals working in general medical practice. Extraintestinal symptomatology is not a specific manifestation of $C D$, but evidence suggests that such forms make CD diagnosis more difficult [7]. In some, patients may remain undiagnosed for years. We wanted to comment on the problems connected to screening methods as an initial step in case finding.

\section{Materials and Methods}

\subsection{Patients}

This report examines an experience of 9 diagnosed adult CD patients. Informed consent was obtained from all of these participants before evaluation. The group consists of outpatients seen by different specialists with extraintestinal symptoms, evaluated and treated for a long period of time before diagnosis of adult CD.

\subsection{Serum Analysis}

Blood from all patients was collected. For each patients, specialized examinations for the detection of antibodies against transglutaminase (tTG, IgA and tTG, IgG), 
antibodies against endomysium (AEA, IgA) against gliadin (AGA, IgA and AGA, IgG) and intolerance to cow milk (AL) were performed.

\subsection{Biopsies}

Each patient underwent between one to two duodenal biopsies and the samples were fixed in a $10 \%$ formalin solution. The samples were hematoxylin \& eosin (H\&E) stained and evaluated in accordance with the MarshOberhuber system [8,9].

\section{Results}

The results are shown in Table 1. The results indicate that IgA antibodies to tTG are a very good indicator of untreated CD. All patients had very high quantitative values of IgA antibodies to tTG. Similar positive results were noted for IgA anti-endomysial antibodies in our hospital. Interestingly, in our limited experience, IgG antibodies to tTG were always negative, indicating that this antibody may not to be suitable as a case finding test for untreated adult $\mathrm{CD}$, at least in our group of adults with atypical clinical presentations, but normal immunoglobulins. Autoantibodies to gliadin showed more variable individual patient results compared with tTG antibodies. The AL values also varied in this series of adult CD patients with elevated IgA antibodies to tTG. In all patients, histological findings documented moderate to severe architectural changes and significant infiltration with intraepithelial lymphocytes.

\begin{tabular}{|c|c|c|c|c|c|c|c|c|c|}
\hline Indicator & Sex & Age & $\begin{array}{l}\text { tTG IgA } \\
\text { (RU/ml) }\end{array}$ & $\begin{array}{l}\text { tTG IgG } \\
\text { (RU/ml) }\end{array}$ & AEA & $\begin{array}{c}\text { AGA IgA } \\
\text { (RU/ml) }\end{array}$ & $\begin{array}{c}\text { AGA IgG } \\
\text { (RU/ml) }\end{array}$ & $\begin{array}{c}\mathrm{AL} \\
\text { (IU/ml) }\end{array}$ & Histology \\
\hline 1 & $\mathrm{~F}$ & 60 & $>200$ & 72.80 & positive & $>200$ & 67.64 & 1.188 & Marsh IIIB \\
\hline 2 & $\mathrm{~F}$ & 41 & $>200$ & 106.20 & positive & $>200$ & $>200$ & $<0.350$ & Marsh IIIB \\
\hline 3 & $\mathrm{~F}$ & 40 & 62.390 & 54.98 & positive & 40.87 & 28.014 & $<0.350$ & Marsh II \\
\hline 4 & $\mathrm{~F}$ & 38 & $>200$ & 98.53 & positive & $>200$ & $>200$ & $<0.350$ & Marsh IIIC \\
\hline 5 & $\mathrm{~F}$ & 75 & $>200$ & 91.60 & positive & 73.323 & 75.179 & $<0.350$ & Marsh IIIC \\
\hline 6 & $\mathrm{~F}$ & 42 & $>200$ & 108.35 & positive & $>200$ & $>200$ & $<0.350$ & Marsh IIIC \\
\hline 7 & $\mathrm{~F}$ & 45 & $>200$ & 98.72 & positive & 84.965 & 96.254 & $<0.350$ & Marsh IIIC \\
\hline 8 & M & 33 & $>200$ & 112.60 & positive & $>200$ & $>200$ & $<0.350$ & Marsh IIIC \\
\hline 9 & M & 20 & $>200$ & 114.50 & positive & 94.266 & 102.969 & $<0.350$ & Marsh IIIC \\
\hline
\end{tabular}

Legend: tTG - autoantibody to tissue transglutaminase, AEA - autoantibody to endomysium, AGA -autoantibody to gliadin, AL - antibody to cow milk, F - woman, $\mathrm{M}$ - man. Internal reference value for tTG, IgA and IgG: positive is more than 150 and for AGA, IgA and IgG: positive is more than 150 .

\section{Discussion}

During the process of case-finding for adult CD, diagnosis depends on a well-constructed search plan in both typical and atypical forms [10]. Several methods of case-finding have been proposed, including several procedures. In practice, intestinal symptoms are usually evident [11,12] although in recent years, atypical presentations of adult CD have become frequently recognized. Varying prevalence of $\mathrm{CD}$ occurs in both children and adults from various states in Europe and other continents. In some of these, results are determined by more intense screening, better education, or even better quality of the diagnostic tools. Targeted screening is an effective exercise that may lead to suspicion for all types of adult CD. But despite the value of seromarkers, there still remain a large number of adults with undiagnosed CD [13]. It may therefore be appropriate to inquire whether these tools have been properly deployed in clinical practice.

Part of the answer to this issue is the power of a CD detection method leading to suspicion of CD. In this clinical experience with atypical clinical presentations, some over prolonged time periods, we used several types of autoantibodies in each patient. Independent studies have shown that these have a different specificity and sensitivity. In our experience, antibodies to IgA tTG proved to be good indicators of untreated CD and are therefore also have been recommended in celiac disease screening [14,15]. A number of studies have documented a high predictive value for celiac disease with the IgA tTG assay [16]. Our results provide confirmatory evidence of this finding, but also show that these serological changes provide strong evidence for the characteristic histopathological changes of untreated celiac disease. Recent evidence suggests that detection of all clinical forms of CD may be most effective with a combination of IgA tTG antibodies coupled with autoantibodies against deaminated gliadin (DGP) $[17,18]$. Specialized groups have also supported the value of serological screening in adult CD $[19,20]$.

Also of concern is the risk of adult CD associated with some atypical clinical presentations and human factors associated with detection. In our patients, improved communication between different physician specialist groups may have been optimal. Although our patients eventually showed positive serology, a long period passed before screening tests were done. This group may be an important group to consider for diagnosis, especially in multi-specialty clinics. This circumstance could possibly trigger a debate about whole-nation screening programs. According to us, however, such alternative is not a right way. Firstly, it is not a targeted form of screening, and secondly, everyone then might then screening to cover other disorders, including cardiovascular diseases or cancer. Such programs would be financially extremely costly. Therefore, according to us, the experts with variable specializations should be repeatedly informed about the various and atypical symptoms of this disease 
with the possibility of sending the material straight to the laboratories capable of autoantibodies examination. This is perhaps the most appropriate way. Otherwise there could repeatedly happen that undiagnosed patients would go around an outpatient care among various specialists. An implemented therapy would only mitigate the effects of the primary disease, or would the patients would be treated for life to other secondary disease. Targeted screening is the most suitable alternative to a search for CD. Patients with a positive test might then be consigned to a gastroenterologist to ensure diagnosis and treatment.

\section{Financial Support}

The work was institutionally supported by RVO 68378050, LM2011032 (MEYS) and the project BIOCEV - Biotechnology and Biomedicine Centre of the Academy of Sciences and Charles University (CZ.1.05/1.1.00/02.0109; European Regional Development Fund).

\section{References}

[1] Beres, N.J., Sziksz, E., Vannay, A., Szabo, D., Pap, D., VeresSzekely, A., Arato, A., Szabo, A.J., Veres, G, Role of the microbiome in celiac disease. International Journal of Celiac Disease, 2 (4). 150-153. 2014.

[2] Plank, L, Primary intestinal malignant lymphomas associated with celiakia - A pathologists review. International Journal of Celiac Disease, 3 (2). 59-68. 2015.

[3] Sziksz, E., Voros, P., Veres, G., Fekete, A., Vannay, A,Coeliac disease: from triggering factors to treatment. International Journal of Celiac Disease, 1(1). 9-13.

[4] Svajdler, M., Daum,O., Rychly, B,Diagnosing celiac disease: Role of the pathologists. International Journal of Celiac Disease,2(2). 70-75. 2014.

[5] Catassi, C., Fasano, A,Is this really celiac disease? Pitfalls in diagnosis. Current Gastroenterology Reports,10(5). 466-472. 2008.

[6] Wakim-Fleming, J., Pagadala, M.R., Lemyre, M.S., Lopez, R., Kumaravel, A., Carey, W.D., Zein, N.N,Diagnosis of celiac disease in adults based on serology test results, without smallbowel biopsy. Clinical Gastroenterology and Hepatology,11(5). 511-516. 2013.

[7] Freeman, H.J, Pearls and pitfalls in the diagnosis of adultceliac disease. Canadian Journal of Gastroenterology, 22(3). 73-280. 2008.

[8] Marsh, M.N, Gluten, major histocompatibility complex, and the small intestine: a molecular and immunologic approach to the spectrum of gluten sensitivity (Celiac Sprue). Gastroenterology, 102(1). 330-354. 1992.

[9] Oberhuber, G., Granditsch, G., Vogelsang, H, The histopathology of coeliac disease: time for a stanmdardized report scheme for pathologist. European Journal of Gastroenterology \& Hepatology, 11(10). 1185-1194. 1999.

[10] Samasca, G., Lupan, I., Deleanu, D., Cristea, V., Makovicky, P, Immunological approach of the challenges of the XXI century in celiac disease. International Reviews of Immunology, 33(1). 3-8. 2014.

[11] Lebwohl, B., Genta, R.M., Kapel, R.C., Sheehan, D., Lerner, N.S., Green, P.H., Neugut, A.L., Rundle, A, Procedure volume influences adherence to celiac disease guidelines. European Journal of Gastroenterology \& Hepatology, 25(11). 1273-1278. 2013.

[12] Zevit, N., Shamir, R, Diagnosis of celiac disease: where are we heading after the ESPGHAN 2012 guidelines? Journal of Pediatric Gastroenterology and Nutrition, 59(S1). 3-5. 2014.

[13] Freeman, H.J, Detection of adult celiac disease with duodenal screening biopsies over a 30-year period. Canadian Journal of Gastroenterology, 27(7). 405-408. 2013.

[14] Reeves, G.E., Burns, C., Hall, S.T., Gleeson, M., Lemmert, K., Clancy, R.L, The measurement of IgA and IgG transglutaminase antibodies in celiac disease: a comparison with current diagnostic methods. Pathology,32(3).181-185. 2000.

[15] Scoglio, R., Di Pasquale, G., Pagano, G., Lucanto, M.C., Magazzu, G., Sferlazzas, C, Is intestinal biopsy always needed for diagnosis of celiac disease? American Journal of Gastroenterology, 98(6). 1325-1331.2003.

[16] Hill, P.G., Forsyth, J.M., Semeraro, D., Holmes, G.K, IgA antibodies to human tissue transglutaminase: audit of routine practice confirms high diagnostics accuracy. Scandinavian Journal of Gastroenterology, 39(11).1078-1082. 2004.

[17] Barakaukas, V.E., Lam, G.Y., Estey, M.P, Digesting all the options: Laboratory testing for celiac disease. Critical Reviews in Clinical Laboratory Sciences, 51(6). 358-378. 2014.

[18] Oana, B., Otilia, M,The usefulness of IgA/IgG DGP/tTG screen assai for celiac disease detection among symptomatic and at risk young children. International Journal of Celiac Disease, 1(1). 2326. 2013.

[19] Husby, S., Koletzko, S., Korponay-Szabo, I.R., Mearin, M.L., Phillips, A., Shamir, R., Troncone, R., Giersiepen, K., Branski, D., Catassi, C., Lelgeman, M., Maki, M., Ribes-Konincky, C., Ventura, A., Zimmer, K.P, European society for pediatric gastroenterology, hepatology, and nutrition guidelines for the diagnosis of celiac disease. Journal of Pediatric Gastroenterology and Nutrition, 54(1). 136-160. 2012.

[20] Ludvigsson, J.F., Bai, J.C., Biagi, F., Card, T.R., Ciacci, C., Ciclitira, P.J., Green, P.H., Hadjivassiliou, M., Holdoway, A., van Heel, D.A., Kaukinen, K., Leffler, D.A., Leonard, J.N., Lundin, K.E., McGough, N., Davidson, M., Murray, J.A., Swift, G.L., Walker, M.M., Zingone, F., Sanders, D.S, Diagnosis and management of adult coeliac disease: guidelines from the British Society of Gastroenterology. Gut, 63(8). 1210-1228. 2014. 